\title{
$\checkmark$ Research Square \\ Clinical expression and prognosis of SUV39H1 in diffuse large B-cell lymphoma
}

\section{Yue Zhang}

Zhengzhou University First Affiliated Hospital

\section{Qing Wen}

Zhengzhou University First Affiliated Hospital

\section{Ya-Xin Lei}

Zhengzhou University First Affiliated Hospital

\section{Jing-Jing $\mathrm{Ge}$}

Zhengzhou University First Affiliated Hospital

\section{Xiao-Shuang Kong}

Zhengzhou University First Affiliated Hospital

\section{Wen-Hua Wang}

Zhengzhou University First Affiliated Hospital

\section{Ze-Yuan Wang}

Zhengzhou University First Affiliated Hospital

\section{Hu-Ting Hou}

Zhengzhou University First Affiliated Hospital

\section{Si-Yu Qian}

Zhengzhou University First Affiliated Hospital

\section{Shao-Xuan Wu}

Zhengzhou University First Affiliated Hospital

\section{Guan-Nan Wang}

Zhengzhou University First Affiliated Hospital

\section{Wen-Cai Li}

Zhengzhou University First Affiliated Hospital

\section{Ming-Zhi Zhang}

Zhengzhou University First Affiliated Hospital

\section{Xu-Dong Zhang}

Zhengzhou University First Affiliated Hospital

Qing-Jiang Chen ( $\nabla$ qingjiang_c@126.com )

Zhengzhou University First Affiliated Hospital https://orcid.org/0000-0001-8441-6190

\section{Research}


Keywords: Diffuse large B-cell lymphoma, SUV39H1, H3K9me3, Prognosis, Epigenetic, Therapeutic targets

Posted Date: April 14th, 2021

DOI: https://doi.org/10.21203/rs.3.rs-418357/v1

License: (1) This work is licensed under a Creative Commons Attribution 4.0 International License. Read Full License 


\section{Abstract}

Background: Epigenetic regulation plays vital roles in the oncogenesis and treatment of diffuse large Bcell lymphoma (DLBCL). The H3K9me3-specific histone methyltransferase SUV39H1 is an epigenetic gene that promotes the progression of a variety of malignancies. However, the roles of SUV39H1 in DLBCL remain unclear.

Methods: Initially, the Oncomine, Cancer Cell Line Encyclopedia (CCLE), UALCAN and Gene Expression Profiling Interactive Analysis (GEPIA) databases were searched to explore the expression of SUV39H1 in DLBCL. The clinical parameters and pathological sections of 61 successive patients, including 47 cases of DLBCL and 14 cases of reactive lymphoid hyperplasia, were collected from January 2019 to November 2020. Immunohistochemistry was conducted to verify the results of the database search. Finally, relevant parameters and pathological results were combined to analyze the expression of SUV39H1.

Results: We found that the expression of SUV39H1 in DLBCL tissues was higher than that in normal and other cancer tissues $(P<0.05)$ in database and immunohistochemistry analyses. Among the analyzed clinical parameters, only tumor size was closely associated with SUV39H1 $(P=0.037)$, suggesting that patients with high SUV39H1 expression are less likely to develop tumors over $7.5 \mathrm{~cm}$ in size. Regarding survival, the group with high SUV39H1 expression had a lower survival rate than the group with low SUV39H1 expression in terms of 10-year disease-free survival (DFS) according to the database analyses $(P=0.035)$. However, SUV39H1 was revealed as an independent predictor of overall survival (OS) and progression-free survival (PFS) in patients from both databases and our hospital $(P>0.05)$.

Conclusion: SUV39H1 expression is higher in DLBCL tissues than in normal and other cancer tissues, indicating that DLBCL patients may not develop bulky tumors over follow-up and suggesting that SUV39H1 might serve not only as a predictive factor in the clinic but also as a target for the epigenetic therapy of DLBCL.

\section{Background}

Diffuse large B-cell lymphoma (DLBCL) is the most common type of non-Hodgkin lymphoma $(\mathrm{NHL})^{[1]}$ and accounts for $30 \%-40 \%$ of all NHLs. According to the HANS classification in 2016, DLBCL can be divided into two types: germinal center B cell-like (GCB) and non-germinal center B cell-like (non-GCB) ${ }^{[2]}$. Its clinical symptoms, immunotypes, histomorphology and genetics are highly heterogenous. Currently, the frontline standard of care for DLBCL is rituximab, cyclophosphamide, doxorubicin, vincristine, and prednisone $(\mathrm{R}-\mathrm{CHOP})^{[3]}$. However, $30 \%-40 \%$ of patients become resistant to drugs or relapse after treatment, and their diseases are associated with rapid progression and a poor prognosis. The 5-year overall survival (OS) rate is $60-70 \%$. The occurrence and development of DLBCL are attributed to the abnormal expression of multiple genes and posttranscriptional regulation ${ }^{[4]}$. Recently, targeted drug therapies aimed at genes involved in the development of DLBCL have achieved success. Thus, further study of DLBCL pathogenesis will play a significant role in providing personalized treatment ${ }^{[5]}$. 
SUV $39 \mathrm{H} 1$ is a member of the SET gene family and functions as a histone methyltransferase to catalyze the methylation of histone $\mathrm{H} 3$ lysine 9 ( $\mathrm{H} 3 \mathrm{~K} 9 \mathrm{me})$ and generate a specific binding site called heterochromatin protein 1 (HP1). HP1 recruits transcription inhibitors, which leads to heterochromatin transcription silencing ${ }^{[6,7]}$. Therefore, the SUV $39 \mathrm{H} 1$ protein is a modified histone enzyme that is able to alter the structure of chromosomes ${ }^{[8]}$ and then affect gene expression. These results suggest that dysfunction of the SUV39H1 protein may trigger the malignant transformation and proliferation of cells, as well as tumorigenesis ${ }^{[9]}$.

Some studies have shown that SUV39H1 is highly expressed and promotes tumor development in many types of carcinomas, such as gastric cancer ${ }^{[10]}$, liver cancer ${ }^{[11]}$, colon cancer ${ }^{[12]}$, and breast cancer ${ }^{[13]}$. However, in DLBCL, the expression of SUV39H1 has not been explicitly reported in most articles. Therefore, in our study, we first searched for information on the differential expressional of SUV39H1 in DLBCL from the Oncomine, the Cancer Cell Line Encyclopedia (CCLE), UALCAN and Gene Expression Profiling Interactive Analysis (GEPIA) databases. Then, we conducted immunohistochemistry assays to verify the results of the databases. Finally, the significance of clinical parameters and the prognosis of SUV39H1 were analyzed to explore new and potent targets to improve the cure rate of DLBCL.

\section{Methods}

\section{Database analyses}

Oncomine (https://www.oncomine.org/resource/login.html) is an online cancer microarray database that integrates RNA and DNA-Seq data from the Gene Expression Omnibus (GEO), The Cancer Genome Atlas (TCGA) and published literature and incorporates 715 datasets and 86,733 samples. It was created by physicians, scientists, and software engineers from Michigan University.

Gene Expression Profiling Interactive Analysis (GEPIA, http://gepia.cancer-pku.cn/) is an interactive web server used to analyze the expression of RNA sequences, including those of 9,736 tumor samples and 8,587 normal samples from the TCGA and Genotype-Tissue Expression (GTEx) databases; it was developed by Zefang Tang et al. at Peking University ${ }^{[14]}$.

The Cancer Cell Line Encyclopedia (CCLE, https://portals.broadinstitute.org/ccle/) is a genomic and pharmacologic web server used to analyze human cancer models. It also provides visualization of genetic data and public access to t over 1100 cell lines; it was conceived by the Broad Institute, the Novartis Institutes for Biomedical Research and the Genomics Institute of the Novartis Research Foundation ${ }^{[15,16]}$.

UALCAN (http://ualcan.path.uab.edu/) is a public database used to analyze TCGA data, identify biomarkers, provide plots depicting protein-coding, miRNA-coding and lincRNA-coding genes, evaluate epigenetic information and select genes/targets by connecting to GeneCards, PubMed, TargetScan, the 
Human Protein Atlas and so on; it was developed by Chandrashekar DS, et al. from the University of Alabama at Birmingham, Birmingham ${ }^{[17]}$.

We retrieved information on the differential expression of SUV39H1 in DLBCL from the Oncomine, GEPIA, CCLE, and UALCAN databases. All the database information on the differential expression of SUV39H1 in DLBCL, clinical parameters and prognosis, was analyzed with the four public websites listed above.

\section{Pathological specimens and clinical parameters of patients}

Paraffin-embedded biopsy specimens and clinical parameters of the patients, including age, sex, subtype, tumor size, lymph node involvement, number of extranodal lesions, Ki-67 index, Ann Arbor stage, B symptoms, eastern cooperative oncology group (ECOG) performance status, lactate dehydrogenase (LDH) level, albumin level, hemoglobin level, procalcitonin level, c-reactive protein level, creatine kinase level, beta-microglobulin level, adenosine deaminase level, international prognostic index (IPI), therapeutic regimen, treatment response, overall survival and progression-free survival times, treated at the First Affiliated Hospital of Zhengzhou University between 2019.01.01 and 2020.11.01, including 47 cases of DLBCL and 14 cases of reactive lymphoid hyperplasia, were collected. All paraffin specimens were diagnosed by two experienced pathologists in our hospital.

\section{Immunohistochemistry (IHC)}

All the DLBCL tissues were embedded in paraffin and cut into $5 \mu \mathrm{m}$ slices. After heating at $55^{\circ} \mathrm{C}$ for deparaffinization, we dewaxed the slices with xylene three times (20 min each time) and rehydrated them with absolute ethanol for $10 \mathrm{~min}, 95 \%$ ethanol for $5 \mathrm{~min}$ and $80 \%$ ethanol for $5 \mathrm{~min}$. Then, we rinsed the slices with Aqua Pura 3 times. Afterwards, slices were incubated in $3 \% \mathrm{H}_{2} \mathrm{O}_{2}$ deionized water at room temperature for 10 min, and a primary antibody against SUV39H1 (1:200; Affinity Biosciences Cat\# DF8452, RRID: AB_2841692) was added. and incubated at $4{ }^{\circ} \mathrm{C}$ overnight. For a negative control, phosphate-buffered saline was prepared instead of the primary antibody. For a positive control, the nuclei were stained brown. The sections were incubated with the secondary antibody for 30 min, treated with an ingraining agent, and sealed with neutral gum. All the images were captured with a digital pathology section scanner (NanoZoomer S210, Hamamatsu Photonics Trading Co. Ltd.) and analyzed with section browsing software (ZYFViewer, version 1.0, Shandong Winmedic Scientific, Inc.).

\section{Grading criteria}

The nuclei of positive cells appeared yellow to brown in color. Five nonrepetitive visual fields were selected under high magnification. At least 200 positive cells per field of vision were counted. Based on the depth of positive staining, we graded the sections as follows: 0 points, no color; 1 point, pale yellow; 2 points, brown; and 3 points, tan. The amount of staining was graded as follows: 0 points, $0-10 \%$ positive cells; 1 point, $11-25 \%$ positive cells; 2 points, $25-50 \%$ positive cells; and 3 points, $>50 \%$ positive cells. The collected images were evaluated by two independent observers, and the above scores from the two 
evaluations were multiplied. Samples with 0-2 points were classified as the low expression group, and those with $\geq 3$ points were classified as the high expression group.

\section{Statistical analysis}

All the databases used (Oncomine, GEPIA, CCLE and UALCAN) are available online. To analyze expression differences between DLBCL and normal tissues, Student's t-test was used to analyze data from the Oncomine database, and one-way ANOVA was used to analyze data from the GEPIA database. The $\chi^{2}$ test was used to determine differences in the expression of SUV39H1 between DLBCL and reactive lymphoid hyperplasia tissues in pathology results. To evaluate the correlations between SUV39H1 and clinical characteristics, Student's t-test was used to analyze data from the UALCAN database, and Fisher's exact test or the $\chi^{2}$ test was used to analyze data from our hospital. The complete response (CR) rate, partial response (PR) rate, and objective response rate (ORR) were also calculated by Fisher's exact test or the $\chi^{2}$ test. OS and PFS were analyzed by Kaplan-Meier curves and compared by the log-rank test. SPSS 21.0 software was used to analyze all the data, and values of $P<0.05$ were considered statistically significant. OS and PFS curves of patients in our hospital were generated with GraphPad Prism 8 software.

\section{Results}

\section{Differential expression of SUV39H1 in DLBCL tissuesb}

By searching the Oncomine database, we found that the expression of SUV39H1 was significantly higher in DLBCL tissues $(P=0.006)$ than in normal nonlymphoid tissues (skin and tonsillar tissues) in Storz dataset ${ }^{[18]}$ (Fig. 1a). According to Compagno dataset ${ }^{[19]}$, SUV39H1 expression is also higher in activated B-cell-like diffuse large $B$ cell lymphoma $(A B C)$ tissues (Fig. 1b, $P=0.010$ ) than in normal lymphoid tissues (germinal center $\mathrm{B}$ lymphocytes, memory $\mathrm{B}$ lymphocytes, and naive pregerminal center $\mathrm{B}$ lymphocytes). These results were validated in the GEPIA database $(P=0.010)$ by comparing 47 DLBCL tissues and 337 normal tissues (Fig. 1C). Moreover, in the GEPIA database, we also discovered that the expression of SUV39H1 was higher in DLBCL than in 30 other types of cancer (Fig. 1d and 1e).

To verify the expression of SUV39H1 in DLBCL and normal lymphoid tissues in the databases, we collected 47 DLBCL pathological sections and 14 reactive lymphoid hyperplasia sections.

Immunohistochemical results showed that in 47 DLBCL tissues, the SUV39H1 protein was mainly stained in the nucleus: 33 samples had high SUV39H1 expression, and 14 samples had low SUV39H1 expression (Fig. 1f, g). The positive rate of SUV39H1 in DLBCL reached up to $70.21 \%$. Among 14 cases of reactive lymphoid hyperplasia, 9 had low SUV39H1 expression, and 5 had high SUV39H1 expression. The positive rate in the control group was only $35.71 \%$. The expression of SUV39H1 in DLBCL tumor tissues was significantly higher than that in reactive lymphoid hyperplasia tissues $(P=0.019)$ (Table 1).

Table 1 Expression differences between DLBCL and reactive lymphoid hyperplasia 


\begin{tabular}{llllll}
\hline Disease type & Total & High (\%) & Low (\%) & $\chi 2$ & $P$ value \\
\hline Diffuse large B-cell lymphoma & 47 & $33(70.21 \%)$ & $14(29.79 \%)$ & $5.466 \quad 0.019 *$ \\
Reactive lymphoid hyperplasia & 14 & $5(35.71 \%)$ & $9(64.29 \%)$ & & \\
\hline
\end{tabular}

$* P<0.05$.

\section{SUV39H1 mRNA expression in DLBCL cell lines}

By searching the CCLE database, we discovered that the mRNA expression of SUV39H1 was higher in DLBCL cell lines than in other malignant tumor cell lines (RNA-seq) (Fig. 2a). The same result was also obtained with the Affymetrix gene chip (Affy), which includes another mRNA expression dataset from the CCLE database (Fig. 2b). Furthermore, after SUV39H1 was knocked down, its expression in the DLBCL cell lines was lower than that in the other malignant tumor cell lines (Fig. 2C). The epigenetic modification (DNA methylation) of SUV39H1 causes SUV39H1 to be expressed at lower levels in DLBCL than in other cancers (Fig. 2d).

\section{Relation between the mRNA level of SUV39H1 and clinicopathological parameters of patients with DLBCL}

With the UALCAN database, we aimed to discover a link between SUV39H1 and clinicopathological parameters. However, we found no obvious differences (Fig. $3, P>0.05$ ), indicating that SUV39H1 is not correlated with tumor stage, sex, age, race, or weight in DLBCL patients.

In the verification of the pathological sections, DLBCL patients were divided into two groups according to immunohistochemical outcomes: SUV39H1 high/low expression groups. We analyzed the relations between SUV39H1 and clinical characteristics in DLBCL patients (Table 2). Between the two groups, there were no significant differences in age, sex, subtype, lymph node invasion, number of extranodal lesions, Ann Arbor stage, Ki-67 index, B symptoms, ECOG performance status, lactate dehydrogenase (LDH) level, $\mathrm{IPI}$, therapeutic regimen, treatment response or other items. However, there was a significant difference in tumor size, suggesting that patients with high expression of the SUV39H1 protein do not develop bulky tumors over $7.5 \mathrm{~cm}$ in size (Table $2, P=0.037$ ).

Table 2 Relations between SUV39H1 and the clinical characteristics of 47 patients with DLBCL 


\begin{tabular}{|c|c|c|c|c|c|}
\hline Clinical parameter & Variable & Total & High (\%) & Low (\%) & $P$ value \\
\hline \multirow[t]{2}{*}{ Age (years) } & $<60$ & 34 & 25 & 9 & 0.486 \\
\hline & $\geq 60$ & 13 & 8 & 5 & \\
\hline \multirow[t]{2}{*}{ Sex } & Male & 22 & 16 & 6 & 0.724 \\
\hline & Female & 25 & 17 & 8 & \\
\hline \multirow[t]{2}{*}{ Subtype } & GCB & 24 & 18 & 6 & 0.464 \\
\hline & Non-GCB & 23 & 15 & 8 & \\
\hline \multirow[t]{2}{*}{ Tumor size } & $<7.5 \mathrm{~cm}$ & 32 & 26 & 6 & $0.037 *$ \\
\hline & $\geq 7.5 \mathrm{~cm}$ & 15 & 7 & 8 & \\
\hline \multirow[t]{2}{*}{ Lymph node involvement } & Involvement & 32 & 20 & 12 & 0.170 \\
\hline & No involvemen & $\mathrm{t} 15$ & 13 & 2 & \\
\hline \multirow[t]{2}{*}{ Number of extranodal lesions } & Involvement & 42 & 30 & 12 & 0.627 \\
\hline & No involvemen & & 3 & 2 & \\
\hline \multirow[t]{2}{*}{ Ki-67 index } & $<60 \%$ & 4 & 3 & 1 & 1.000 \\
\hline & $\geq 60 \%$ & 43 & 30 & 13 & \\
\hline \multirow[t]{2}{*}{ Ann Arbor stage } & I-II & 32 & 23 & 9 & 0.742 \\
\hline & III-IV & 15 & 10 & 5 & \\
\hline \multirow[t]{2}{*}{ B symptoms } & Present & 5 & 5 & 0 & 0.303 \\
\hline & Absent & 42 & 28 & 14 & \\
\hline \multirow[t]{2}{*}{ ECOG performance status } & $0-1$ & 32 & 22 & 10 & 1.000 \\
\hline & $2-5$ & 15 & 11 & 4 & \\
\hline \multirow[t]{3}{*}{ LDH level } & Elevated & 21 & 14 & 7 & 0.335 \\
\hline & Normal & 20 & 16 & 4 & \\
\hline & Undetected & 6 & 3 & 3 & \\
\hline \multirow[t]{2}{*}{ Albumin level } & Low & 3 & 2 & 1 & 1.000 \\
\hline & Normal & 44 & 21 & 13 & \\
\hline \multirow[t]{2}{*}{ Hemoglobin level } & Low & 29 & 20 & 9 & 0.812 \\
\hline & Normal & 18 & 13 & 5 & \\
\hline \multirow[t]{3}{*}{ Procalcitonin (PCT) level } & Elevated & 14 & 12 & 2 & 0.385 \\
\hline & Normal & 13 & 9 & 4 & \\
\hline & Undetected & 20 & 12 & 8 & \\
\hline C-reactive protein level & Elevated & 18 & 13 & 5 & 1.000 \\
\hline
\end{tabular}




\begin{tabular}{llllll} 
& Normal & 13 & 9 & 4 & \\
Creatine kinase (CK) level & Undetected & 16 & 11 & 5 & \\
& Low & 9 & 8 & 1 & 0.210 \\
& Normal & 23 & 14 & 9 & \\
Beta-microglobulin level & Elevated & 6 & 5 & 1 & 1.000 \\
& Normal & 35 & 25 & 10 & \\
Adenosine deaminase level & Undetected & 6 & 3 & 3 & \\
& Elevated & 9 & 7 & 2 & 1.000 \\
& Normal & 23 & 16 & 7 & \\
IPI & Undetected & 15 & 10 & 5 & \\
Therapeutic regimen & 0-2 & 32 & 22 & 10 & 1.000 \\
& C-5 & 15 & 11 & 4 & \\
& Chemotherapy & 43 & 30 & 13 & 1.000 \\
& Radiotherapy & 0 & 0 & 0 & \\
Treatment response & Combined & 4 & 3 & 1 & \\
& CR』PR & 38 & 27 & 10 & 0.456 \\
& SDaPD & 9 & 6 & 4 & \\
\hline
\end{tabular}

Abbreviations: ECOG, Eastern Cooperative Oncology Group; LDH, lactate dehydrogenase; CR, complete response; PR, partial response; PD, progressive disease; SD, stable disease; IPI, International Prognostic Index. $* P<0.05$.

\section{The prognostic value of SUV39H1 in DLBCL}

We calculated the CR rate, $\mathrm{PR}$ rate and ORR of DLBCL patients who were treated only with R-CHOP in the SUV39H1 high- and low-expression groups to explore the associations between SUV39H1 and short-term prognosis. Among these patients, only 26 received the R-CHOP therapeutic regimen: 19 patients with high SUV39H1 expression and 7 patients with low SUV39H1 expression. Specifically, in the group with high SUV39H1 expression, 12 patients achieved $\mathrm{CR}$, 7 patients achieved $\mathrm{PR}$, and 19 patients achieved objective response. In the group with low SUV39H1 expression, 4 patients achieved CR, 3 patients achieved PR and 7 patients achieved objective response. However, none of the parameters described above were significant (Table $3, P>0.05$ ).

Table 3 Response rates of the SUV39H1 high/low expression groups 


\begin{tabular}{lccc}
\hline Response & \multicolumn{2}{c}{ Number of patients (\%) } & $P$ value \\
& High $(\mathrm{N}=33)$ & Low $(\mathrm{N}=14)$ & \\
\hline CR & $12(36.4)$ & $4(28.6)$ & 0.742 \\
PR & $7(21.2)$ & $3(21.4)$ & 1.000 \\
ORR & $19(57.6)$ & $7(50.0)$ & 0.633 \\
\hline
\end{tabular}

Abbreviations: $\mathrm{CR}$, complete response; $\mathrm{PR}$, partial response; ORR, objective response rate; $* P<0.05$.

In the GEPIA database, we obtained OS and DFS data according to SUV39H1 expression in DLBCL patients (Fig. 4a). When the high and low SUV39H1 expression groups were compared, the 16-year DFS time and rate of the high SUV39H1 expression group were significantly lower than those of the low SUV39H1 expression group $(P=0.035)$. In terms of 16 -year OS, the survival time was markedly shortened, but the survival rate was not significantly different $(P=0.32)$. The OS/PFS outcomes were also analyzed in 47 patients from our hospital (Fig. 4b). The results revealed no obvious differences in OS/PFS between the high and low SUV39H1 expression groups $(P>0.05)$.

We also performed univariate analysis to investigate the relationship between SUV39H1 and OS/PFS. The results revealed that SUV39H1 was independent of OS and PFS in DLBCL patients. Similar results were also obtained for other factors, such as age, sex, subtype, lymph node invasion, Ann Arbor stage, B symptoms, ECOG performance status, LDH level, hemoglobin level, IPI, therapeutic regimen, and tumor size. However, the albumin level was significant for the OS $(P=0.004)$ and PFS $(P=0.020)$ of DLBCL patients, illustrating that albumin is associated with the OS and PFS of DLBCL patients independent of SUV39H1 (Table 4).

Table 4 Univariate analysis of overall survival and progression-free survival among DLBCL patients 
Parameter
Overall

survival

$\mathrm{P}$ value

SUV39H1 expression

(high vs. low)

Age (years)

(<60 vs. $\geq 60$ )

Sex

(male vs. female)

Ann Arbor stage

(III/IV vs. I/II)

B symptoms

(present vs. absent)

ECOG performance status

(2-5 vs. 0-1)

LDH level

(elevated vs. normal)

Hemoglobin level

(low vs. normal)

Albumin level

(low vs. normal)

IPI

(2-3 vs. $0-1)$

Lymph node involvement

(involvement vs. no involvement)

Therapy regimen

(chemo vs. radio vs. combination)

Tumor size

$(<5$ vs. $\geq 5 \mathrm{~cm})$
0.404

$0.004 *$

$0.020 *$

0.835

0.893

0.572

0.667

Progression-free

survival

$P$ value

0.814

0.147

0.129

0.862

0.485

0.224

0.645

0.802

0.333

0.267

0.221

Abbreviations: ECOG, Eastern Cooperative Oncology Group; LDH, lactate dehydrogenase; IPI, International Prognostic Index; ${ }^{*} P<0.05$. 


\section{Discussion}

Previous studies have shown that SUV39H1 regulates cyclin E by interacting with the retinoblastoma $(\mathrm{Rb})$ protein. Cyclin $\mathrm{E}$ is an important regulator of the $\mathrm{G} 1 / \mathrm{S}$ phase of the cell cycle ${ }^{[6,20]}$. The Rb protein interacts with the SUV39H1 protein to reshape the structure of chromatin and inhibit transcription. Therefore, the functional loss of Rb may induce cell cycle disorders, resulting in a malignant phenotype. That is, all the factors that destroy the interaction of $\mathrm{SUV} 39 \mathrm{H} 1$ and $\mathrm{Rb}$ might play important roles in tumorigenesis ${ }^{[21]}$. These results were also validated in specific cancers. For instance, in human melanoma, Kim G et al. found that the SUV39H1-dependent Rb promoter has a positive correlation with a target gene and leads to tumor formation and development ${ }^{[22]}$. Upon the exploration of more effective approaches for breast cancer therapy, similar outcomes also illustrated that both SUV39H1 and Rb regulate a transcription repressor complex ${ }^{[23]}$.

SUV39H1 is also associated with cell senescence and apoptosis ${ }^{[24]}$. The dephosphorylation of p53 intensifies the subsequent stability of SUV39H1, which is one of the critical features in cellular senescence $^{[25]}$. The relevant mechanism of senescence also correlates with the TGF-beta-related microenvironment ${ }^{[26]}$. Recently, Milanovic M et al. discovered that SUV39H1 is a critical signal component in the senescence machinery and functions as a stem cell regulator; this is collectively defined as 'stemness'. This senescence-associated stemness has important significance in cancer aggressiveness and clinical prognosis and is enriched in recurrent cancers ${ }^{[27]}$. We speculated that high SUV39H1 expression may be related to some signal components of stem cells, leading to the progression and aggressiveness of DLBCL. Furthermore, a new target gene called USP7 cooperates with p53 to induce. Sathish Kumar Mungamuri et al. discovered that USP7 occupies p53 target promoters and forms a trimeric complex with MDM2 and SUV39H1. Then, the complex regulates the ubiquitination of MDM2dependent SUV39H1 and induces apoptosis ${ }^{[28]}$.

In most solid tumors, SUV39H1 expression is high, but its expression in cervical cancer is low. Chiba et al. found that patients with high SUV39H1 expression had a significantly high rate of hepatocellular carcinoma recurrence ${ }^{[11]}$. In gastric cancer, Cai et al. illustrated that SUV39H1 could induce the demethylation of miR-125a-5p, which suppresses the ability of miR-125a-5p to inhibit cancer invasion and progression. Blocking SUV39H1 might be a potential therapeutic target in gastric carcinoma ${ }^{[10,29]}$. SUV39H1 was found to be upregulated in colorectal carcinoma (CRC) tissues compared to normal colon tissues in the tumor microenvironment to promote immune escape. In addition, the small molecule inhibitor F5446 is aimed at SUV39H1. The SUV39H1-selective inhibitor F5446 can transform the functions of known genes involved in DNA replication at $S$ phase and increase the sensitivity of CRC cells to apoptosis in vitro and in vivo ${ }^{[12,30]}$. In prostate cancer, it was reported that the elevated expression of SUV39H1 promotes cancer cell aggression ${ }^{[31]}$. In breast cancer, SUV39H1 binds to PHACTR2-AS1-30ntRNA, silences DNA genes and then restrains breast cancer progression and lung metastasis in mice ${ }^{[32]}$. In 
malignant brain glioma, tumors can be suppressed by repressive targets of $\mathrm{SUV} 39 \mathrm{H} 1^{[33]}$. However, in cervical cancer, the weak chromatin state of SUV39H1 promotes cell migration; thus, SUV39H1 might be a novel and critical target for epigenetic therapies ${ }^{[34]}$. In our study, we found that SUV39H1 expression was higher in DLBCL than in other kinds of solid tumors regardless of the tissue or cell type by searching the GEPIA and CCLE databases and verifying our results pathologically. These results suggest that SUV39H1 may be a potential target for the epigenetic therapy of DLBCL in the future.

Previous studies have reported that SUV39H1 is a cancer suppressor gene in hematological malignancies and lymphomas. The H3K9-mediated senescence mechanism depends on the ability of SUV39H1 to inhibit the formation and progression of primary lymphocytes. Therefore, SUV39H1-deficient lymphoma cells usually grow rapidly ${ }^{[35]}$. Moreover, in T lymphoblastoid and monocytic cell lines that are chronically infected by HIV-1, methyltransferase SUV39H1 acts on the HIV-1 promoter, increasing the transcription of HIV-1 and promoting apoptosis ${ }^{[36]}$. Some researchers have found in the E $\mu$-myc transgenic mouse lymphoma model that therapy-induced senescence (TIS) depends on SUV39H1. TIS-competent lymphomas, which are Suv39H1(+) lymphomas, can increase glucose utilization, produce more ATP and prompt tumor regression ${ }^{[37]}$. However, SUV39H1 also functions as a cancer-promoting gene in lymphoma. Target gene promoters in cutaneous T cell lymphoma (CTCL) can be occluded by H3K9me. It is worth noting that the repression of $\mathrm{SUV} 39 \mathrm{H} 1$ can recover the functions of the target gene and effectively inhibit the growth of Sézary cells ${ }^{[38]}$. In our study, high SUV39H1 expression was an important factor leading to the tumorigenesis and progression of DLBCL. Moreover, some researchers discovered that SUV39H1 plays a vital role in the differentiation of hematopoietic stem cells (HSCs) into B cells. And the expression of SUV39H1 shows an increase in human HSC with the age decrease ${ }^{[39]}$. However, in our study, regardless of the patients analyzed, age had no obvious relationship with the expression of SUV39H1 in DLBCL ( $P>0.05)$. Finally, we conclude that DLBCL patients with high SUV39H1 expression might not develop bulky tumors, which could be a feature of DLBCL patients with high SUV39H1 expression and provide a good index for doctors to evaluate the development of DLBCL.

\section{Conclusion}

Our study confirmed that SUV39H1 expression is higher in DLBCL tissues than in normal and other cancer tissues. This high expression of SUV39H1 is strongly correlated with tumor size, indicating that DLBCL patients may not develop bulky tumors in the course of subsequent treatment. All these discoveries might provide a novel and potential target for the epigenetic therapy of DLBCL and a special indicator for clinicians to evaluate disease development.

\section{Abbreviations}

IHC: Immunohistochemistry; DLBCL: Diffuse large B-cell lymphoma; NHL: Non-Hodgkin lymphoma; GCB: Germinal center B cell-like; Non-GCB: Non-germinal center B cell-like; DFS: Disease-free survival; OS: Overall survival; PFS: Progression-free survival; HP1: Heterochromatin protein 1; R-CHOP: Rituximab, 
cyclophosphamide, doxorubicin, vincristine and prednisone; GEO: Gene Expression Omnibus; TCGA: The Cancer Genome Atlas; GEPIA: Gene Expression Profiling Interactive Analysis; GTEx: Genotype-Tissue Expression; CCLE: Cancer Cell Line Encyclopedia; ECOG: Eastern Cooperative Oncology Group; LDH: Lactate dehydrogenase; IPI: International Prognostic Index; CR: Complete response; PR: Partial response; ORR: Objective response rate; PD: Progressive disease; SD: Stable disease.

\section{Declarations}

\section{Ethics approval and consent to participate}

Not applicable.

\section{Consent for publication}

All the authors have read and consented to submit the article to this journal.

\section{Availability of data and materials}

The datasets supporting the conclusions of this article are available in the Oncomine (https://www.oncomine.org/resource/login.html), Gene Expression Profiling Interactive Analysis (GEPIA, http://gepia.cancer-pku.cn/), Cancer Cell Line Encyclopedia (CCLE, https://portals.broadinstitute.org/ccle/) and UALCAN (http://ualcan.path.uab.edu/) databases.

\section{Competing interests}

All the authors declare that they have no competing interests.

\section{Funding}

This study was supported by the National Natural Science Foundation of China (Grant No. 82070210), Major Medical Scientific and Technological Project of Henan Province (Grant No. SBGJ202001008), and National Science and Technology Major Project of China (Grant No. 2020ZX09201- 009).

\section{Authors' contributions}

YZ, QW, YXL, JJG, WHW, XSK, ZYW, HTH, SYQ, SXW, collected the data. YZ analyzed the data and drafted the manuscript. WCL and GNW provided pathological sections. QJC provided the funding for this study. XDZ, MZZ, and QJC provided administrative and logistical support for this article. All the authors read and approved the final manuscript.

\section{Acknowledgments}

Not applicable. 


\section{References}

[1] Teras LR, DeSantis CE, Cerhan JR, Morton LM, Jemal A, Flowers CR. 2016 US lymphoid malignancy statistics by World Health Organization subtypes. CA Cancer J Clin. 2016. 66(6): 443-459.

[2] Hans CP, Weisenburger DD, Greiner TC, et al. Confirmation of the molecular classification of diffuse large B-cell lymphoma by immunohistochemistry using a tissue microarray. Blood. 2004. 103(1): 275-82.

[3] Xu PP, Fu D, Li JY, et al. Anthracycline dose optimisation in patients with diffuse large B-cell lymphoma: a multicentre, phase 3, randomised, controlled trial. Lancet Haematol. 2019. 6(6): e328-e337.

[4] Li S, Young KH, Medeiros LJ. Diffuse large B-cell lymphoma. Pathology. 2018. 50(1): 74-87.

[5] Nastoupil LJ, Jain MD, Feng L, et al. Standard-of-Care Axicabtagene Ciloleucel for Relapsed or Refractory Large B-Cell Lymphoma: Results From the US Lymphoma CAR T Consortium. J Clin Oncol. 2020. 38(27): 3119-3128.

[6] Nielsen SJ, Schneider R, Bauer UM, et al. Rb targets histone H3 methylation and HP1 to promoters. Nature. 2001. 412(6846): 561-5.

[7] Dodge JE, Kang YK, Beppu H, Lei H, Li E. Histone H3-K9 methyltransferase ESET is essential for early development. Mol Cell Biol. 2004. 24(6): 2478-86.

[8] Peters AH, O\&\#39, Carroll D, et al. Loss of the Suv39h histone methyltransferases impairs mammalian heterochromatin and genome stability. Cell. 2001. 107(3): 323-37.

[9] Antunes E, Ottersbach K. The MLL/SET family and haematopoiesis. Biochim Biophys Acta Gene Regul Mech. 2020. 1863(8): 194579.

[10] Cai M, Chen Q, Shen J, Lv C, Cai L. Epigenetic silenced miR-125a-5p could be self-activated through targeting Suv39H1 in gastric cancer. J Cell Mol Med. 2018. 22(10): 4721-4731.

[11] Chiba T, Saito T, Yuki K, et al. Histone lysine methyltransferase SUV39H1 is a potent target for epigenetic therapy of hepatocellular carcinoma. Int J Cancer. 2015. 136(2): 289-98.

[12] Lu C, Yang D, Klement JD, et al. SUV39H1 Represses the Expression of Cytotoxic T-Lymphocyte Effector Genes to Promote Colon Tumor Immune Evasion. Cancer Immunol Res. 2019. 7(3): 414-427.

[13] Mo W, Liu Q, Lin CC, et al. mTOR Inhibitors Suppress Homologous Recombination Repair and Synergize with PARP Inhibitors via Regulating SUV39H1 in BRCA-Proficient Triple-Negative Breast Cancer. Clin Cancer Res. 2016. 22(7): 1699-712.

[14] Tang Z, Li C, Kang B, Gao G, Li C, Zhang Z. GEPIA: a web server for cancer and normal gene expression profiling and interactive analyses. Nucleic Acids Res. 2017. 45(W1): W98-W102. 
[15] Li H, Ning S, Ghandi M, et al. The landscape of cancer cell line metabolism. Nat Med. 2019. 25(5): 850-860.

[16] Ghandi M, Huang FW, Jané-Valbuena J, et al. Next-generation characterization of the Cancer Cell Line Encyclopedia. Nature. 2019. 569(7757): 503-508.

[17] Chandrashekar DS, Bashel B, Balasubramanya S, et al. UALCAN: A Portal for Facilitating Tumor Subgroup Gene Expression and Survival Analyses. Neoplasia. 2017. 19(8): 649-658.

[18] Storz MN, van de Rijn M, Kim YH, Mraz-Gernhard S, Hoppe RT, Kohler S. Gene expression profiles of cutaneous B cell lymphoma. J Invest Dermatol. 2003. 120(5): 865-70.

[19] Compagno M, Lim WK, Grunn A, et al. Mutations of multiple genes cause deregulation of NFkappaB in diffuse large B-cell lymphoma. Nature. 2009. 459(7247): 717-21.

[20] Hossain M, Stillman B. Opposing roles for DNA replication initiator proteins ORC1 and CDC6 in control of Cyclin E gene transcription. Elife. 2016. 5.

[21] Giacinti C, Giordano A. RB and cell cycle progression. Oncogene. 2006. 25(38): 5220-7.

[22] Kim G, Kim JY, Lim SC, Lee KY, Kim O, Choi HS. SUV39H1/DNMT3A-dependent methylation of the RB1 promoter stimulates PIN1 expression and melanoma development. FASEB J. 2018. 32(10): 56475660.

[23] Li Y, Yuan YY, Meeran SM, Tollefsbol TO. Synergistic epigenetic reactivation of estrogen receptor-a (ERa) by combined green tea polyphenol and histone deacetylase inhibitor in ERa-negative breast cancer cells. Mol Cancer. 2010. 9: 274.

[24] Campaner S, Doni M, Verrecchia A, Fagà G, Bianchi L, Amati B. Myc, Cdk2 and cellular senescence: Old players, new game. Cell Cycle. 2010. 9(18): 3655-61.

[25] Park JW, Bae YS. Dephosphorylation of p53 Ser 392 Enhances Trimethylation of Histone H3 Lys 9 via SUV39h1 Stabilization in CK2 Downregulation-Mediated Senescence. Mol Cells. 2019. 42(11): 773782.

[26] Reimann M, Lee S, Loddenkemper C, et al. Tumor stroma-derived TGF-beta limits myc-driven lymphomagenesis via Suv39h1-dependent senescence. Cancer Cell. 2010. 17(3): 262-72.

[27] Milanovic M, Fan D, Belenki D, et al. Senescence-associated reprogramming promotes cancer stemness. Nature. 2018. 553(7686): 96-100.

[28] Mungamuri SK, Qiao RF, Yao S, Manfredi JJ, Gu W, Aaronson SA. USP7 Enforces Heterochromatinization of $\mathrm{p} 53$ Target Promoters by Protecting SUV39H1 from MDM2-Mediated Degradation. Cell Rep. 2016. 14(11): 2528-37. 
[29] Cai L, Ma X, Huang Y, Zou Y, Chen X. Aberrant histone methylation and the effect of Suv39H1 siRNA on gastric carcinoma. Oncol Rep. 2014. 31(6): 2593-600.

[30] Lu C, Klement JD, Yang D, et al. SUV39H1 regulates human colon carcinoma apoptosis and cell cycle to promote tumor growth. Cancer Lett. 2020. 476: 87-96.

[31] Yu T, Wang C, Yang J, Guo Y, Wu Y, Li X. Metformin inhibits SUV39H1-mediated migration of prostate cancer cells. Oncogenesis. 2017. 6(5): e324.

[32] Chu W, Zhang X, Qi L, et al. The EZH2-PHACTR2-AS1-Ribosome Axis induces Genomic Instability and Promotes Growth and Metastasis in Breast Cancer. Cancer Res. 2020. 80(13): 2737-2750.

[33] Liao HF, Lee CC, Hsiao PC, et al. TCH1036, a indeno[1,2-c]quinoline derivative, potentially inhibited the growth of human brain malignant glioma (GBM) 8401 cells via suppression of the expression of Suv39h1 and PARP. Biomed Pharmacother. 2016. 82: 649-59.

[34] Rodrigues C, Pattabiraman C, Vijaykumar A, et al. A SUV39H1-low chromatin state characterises and promotes migratory properties of cervical cancer cells. Exp Cell Res. 2019. 378(2): 206-216.

[35] Braig M, Lee S, Loddenkemper C, et al. Oncogene-induced senescence as an initial barrier in lymphoma development. Nature. 2005. 436(7051): 660-5.

[36] Iordanskiy S, Van Duyne R, Sampey GC, et al. Therapeutic doses of irradiation activate viral transcription and induce apoptosis in HIV-1 infected cells. Virology. 2015. 485: 1-15.

[37] Dörr JR, Yu Y, Milanovic M, et al. Synthetic lethal metabolic targeting of cellular senescence in cancer therapy. Nature. 2013. 501(7467): 421-5.

[38] Harro CM, Perez-Sanz J, Costich TL, et al. Methyltransferase inhibitors restore SATB1 protective activity against cutaneous T cell lymphoma in mice. J Clin Invest. 2021. 131(3).

[39] Djeghloul D, Kuranda K, Kuzniak I, et al. Age-Associated Decrease of the Histone Methyltransferase SUV39H1 in HSC Perturbs Heterochromatin and B Lymphoid Differentiation. Stem Cell Reports. 2016. 6(6): 970-984.

\section{Figures}



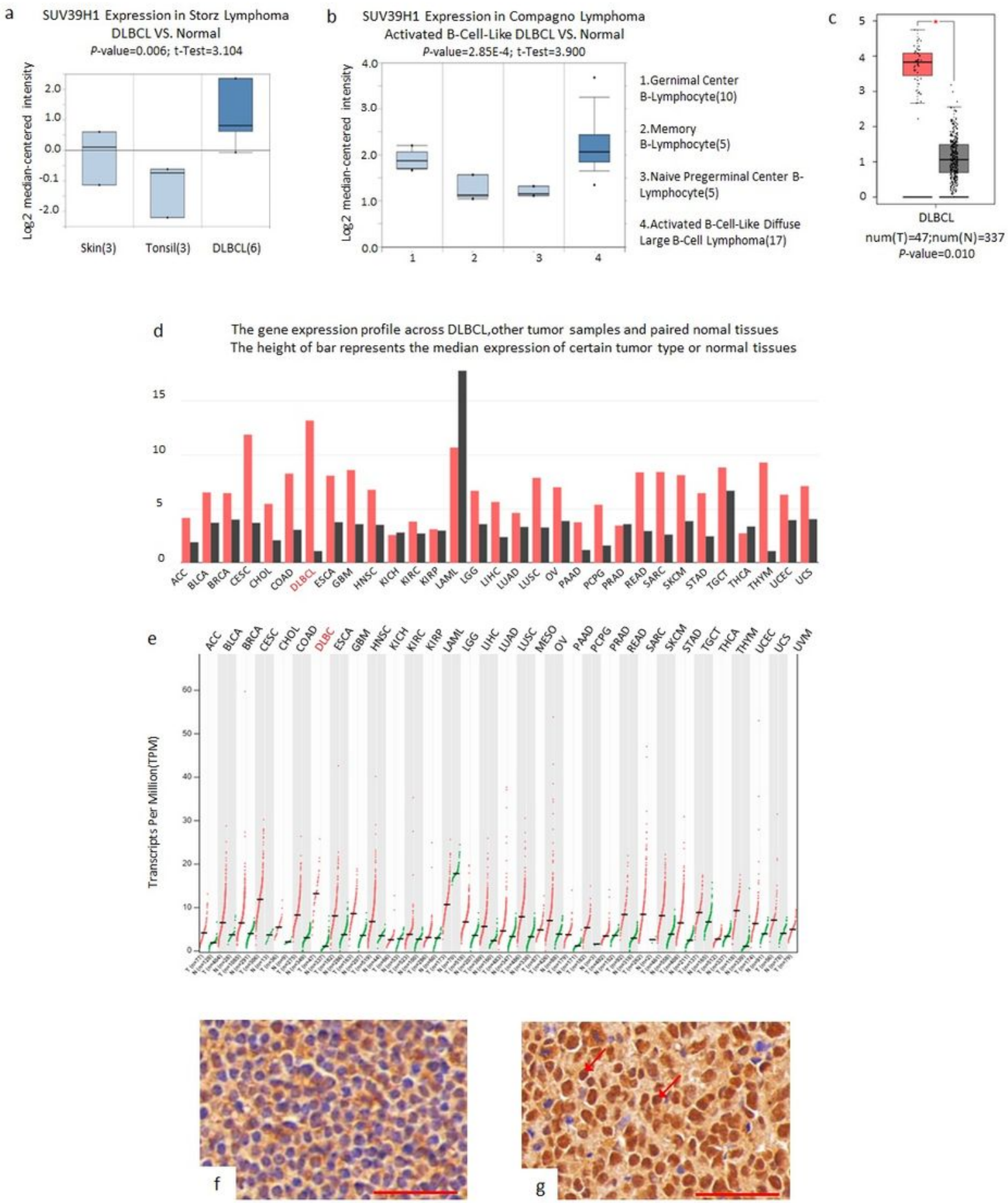

\section{Figure 1}

Differential expression of SUV39H1 in DLBCL and normal tissues. (a), (b), (c) The differential expression of SUV39H1 between DLBCL and normal tissues in the Oncomine and GEPIA databases. (d), (e) The differential expression of SUV39H1 between DLBCL and 30 other types of tumors in the GEPIA database. (f) The expression of SUV39H1 in reactive lymphoid hyperplasia tissue by immunohistochemistry (40x). (g) The expression of SUV39H1 in DLBCL tissue by immunohistochemistry (40x); Scale: $50 \mu \mathrm{m}$. 

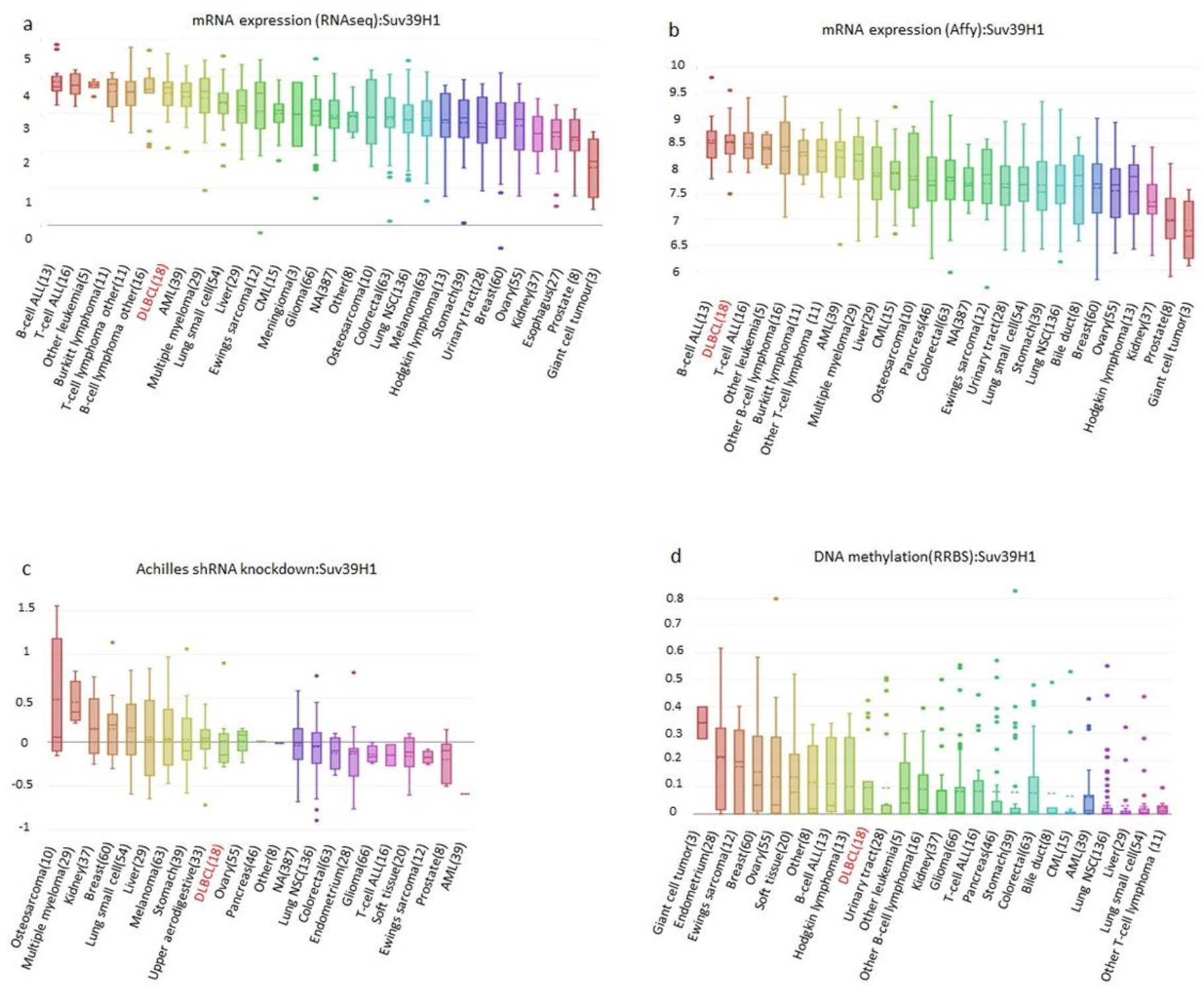

\section{Figure 2}

Expression of SUV39H1 in cell lines from the CCLE database. (a), (b) The mRNA levels of SUV39H1 in DLBCL and other cancers in the RNA sequencing datasets (RNA-seq) and Affymetrix gene chip (affy). (c) Expression differences in SUV39H1 between DLBCL and other tumors in the Achilles dataset after shRNA knockdown. (d) DNA methylation of SUV39H1 in different kinds of malignant tumors in the RRBS dataset. 

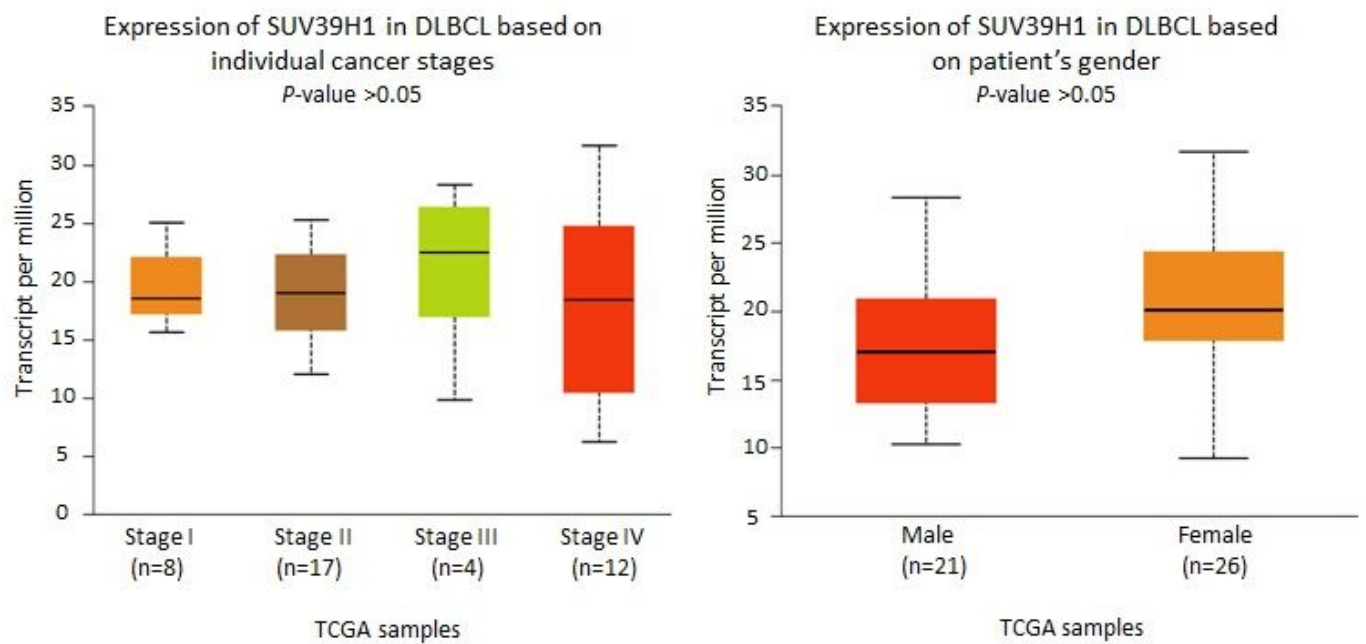

Expression of SUV39H1 in DLBCL based on patient's age $P$-value $>0.05$

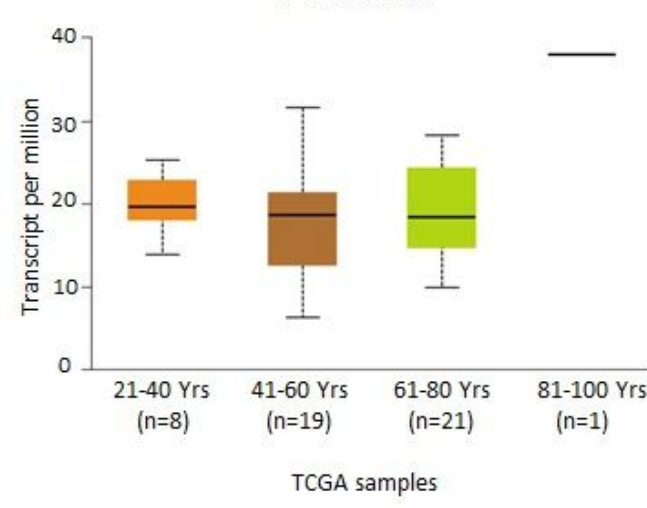

Expression of SUV39H1 in DLBCL based on patient's race $P$-value $>0.05$

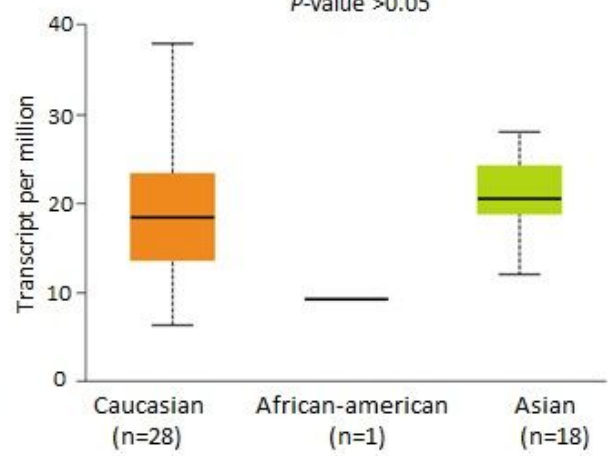

TCGA samples
Expression of SUV39H1 in DLBCL based on patient's weight $P$-value $>0.05$

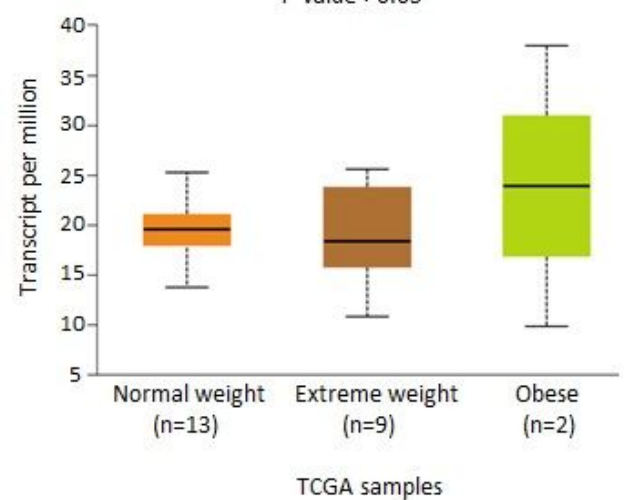

TCGA samples

\section{Figure 3}

Differences in SUV39H1 expression in DLBCL according to different clinicopathological parameters, such as cancer stage, patient sex, age, race, and weight, in the UALCAN database. 

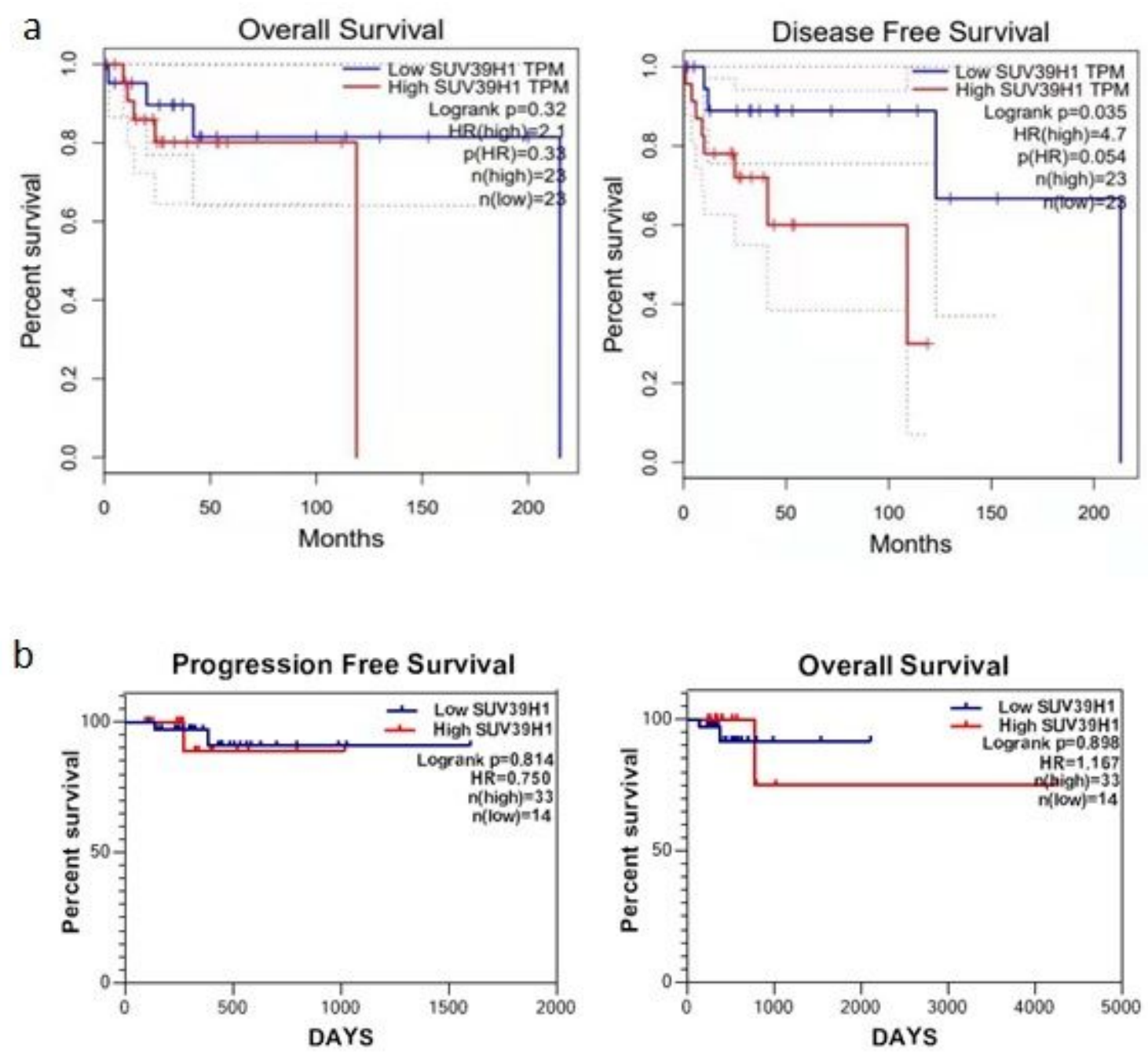

\section{Figure 4}

The relations etween SUV39H1 and long-term prognostic indexes (OS, DFS, and PFS) of DLBCL patients. (a) OS and DFS differences between the high and low SUV39H1 expression groups in the GEPIA database. (b) OS and PFS differences between the high and low SUV39H1 expression groups in patients in our hospital. 